\title{
Zwischen Tradition und Sozialmanagement
}

\section{Einblicke in sozioökonomische und politische Spannungsfelder der freien Wohlfahrt}

Obwohl Wohlfahrtsverbände einen enormen Beitrag zur sozialen Infrastruktur in Deutschland leisten, zentrale Arbeitgeber im Sozial- und Bildungsbereich sind und hohen Einfluss auf sozialpolitische Entscheidungen in einem reichen Land des globalen Nordens nehmen, wurden sie in den letzten Jahren kaum wissenschaftlich beleuchtet. Umstrukturierungen, etwa die Trennung der Idealverbände vom operativen Geschäft, und Expansionen, z. B. in der Pflege und der Bildung, Erziehung und Betreuung von Kindern, verliefen weitgehend unter dem Radar organisationsanalytischer Empirie. Was wissen wir eigentlich über die vielfältigen Strukturen und aktuellen Herausforderungen der Wohlfahrtsverbände?

$\mathrm{D}$ ie sechs staatlich anerkannten Spitzenverbände der Freien Wohlfahrtspflege (im Folgenden kurz „Wohlfahrtsverbände“) in Deutschland die AWO, der Deutsche Caritasverband, das Diakonische Werk der evangelischen Kirchen in Deutschland, der PARTITÄTISCHE Gesamtverband, das Deutsche Rote Kreuz und die Zentralwohlfahrtsstelle der Juden in Deutschland - genießen selbst im internationalen Vergleich der reichen Staaten des globalen Nordens eine gesellschaftliche und rechtliche Sonderstellung, die historisch gewachsen ist und sich auch in ihren ideologisch

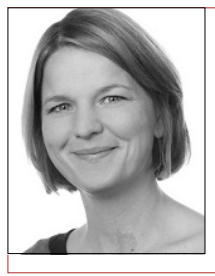

\section{Barbara Lochner}

Fachhochschule Erfurt, Erfurt, Deutschland

*1978, Prof. Dr., Dipl.-Sozialpädagogin, M. A., Professorin für Pädagogik der Kindheit an der Fakultät für angewandte Sozialwissenschaften der Fachhochschule Erfurt.

barbara.lochner@fh-erfurt.de

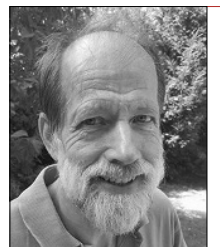

\section{Helmuth Schweitzer}

Duisburg, Deutschland

*1952, Dr., Dipl.-Pädagoge. Zwischen 1973 und 1984 Beschäftigter in Einrichtungen der Caritas, des Diakonischen Werks, der Arbeiterwohlfahrt und des Paritätischen Wohlfahrtverbands. Bis 2017 Leiter des Kommunalen Integrationszentrum der Stadt Essen, nun im (Un)Ruhestand. Seit März 2018 TeilzeitVertretungslehrer an einer Grundschule in Duisburg-Marxloh. Vorsitzender von Laissez-passer e. V. in Essen. helmuth.schweitzer@gmx.de

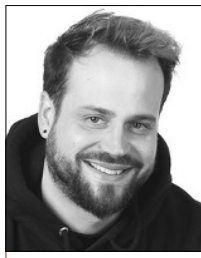

\section{Dirk Kratz}

Landau, Deutschland

*1980, Dr. phil., Diplom-Pädagoge, Psychodrama-Praktiker Geschäftsführer des Therapieverbundes Ludwigsmühle, Vorstandsmitglied im fdr+ Fachverband Drogen- und Suchthilfe e. V.

dirk.kratz@ludwigsmuehle.de
Zusammenfassung Der einleitende Beitrag des Themenschwerpunkts zur aktuellen Situation von Wohlfahrtsverbänden zielt darauf, wesentliche Knotenpunkte der Diskussion zusammenzufassen. Er benennt vor dem Hintergrund der verbandlichen Vielfalt Forschungsdesiderata zu den Auswirkungen verschärfter sozialwirtschaftlicher Rahmenbedingungen auf die Gestaltungsspielräume bei der Sicherung nachhaltiger finanzieller Grundlagen für Existenz und Leistungserbringung der Organisationen.

Schlüsselwörter Wohlfahrtsverbände, Sozialmanagement, Soziale Nachhaltigkeit, Wirtschaftlichkeit

geprägten Traditionen begründet. Diese Sonderstellung, in Deutschland bekräftigt durch das gesetzlich verankerte „Subsidiaritätsprinzip“, nehmen Wohlfahrtsverbände nach wie vor in Anspruch - für sich, aber auch für ihre gemeinnützigen Trägergesellschaften und Mitgliedsorganisationen, die hierzulande mittlerweile einen großen Teil der sozialen Dienstleistungen übernommen und ausgeweitet haben.

Die Entwicklung der Wohlfahrtsverbände ist geprägt durch die Zunahme der Komplexität sozialer Dienstleistungen und damit einhergehenden Professionalisierungserfordernissen. Wesentlich sind dabei eine stärkere Forderung nach Wirtschaftlichkeit, die Auseinandersetzung mit rechtlichen Fragen der Verantwortungsübernahme, wie auch die Frage nach der gesellschaftlichen Verankerung von Wohlfahrt durch Mitgliedschaften, Ehrenamt und politischer Beteiligung. Den damit verbundenen vielschichtigen Anforderungen gerecht zu werden und sie in ihrer Widersprüchlichkeit adäquat zu bedienen, scheint nicht immer zu gelingen: „Eine Reihe von Problemen der Wohlfahrtsverbände wird aus der 

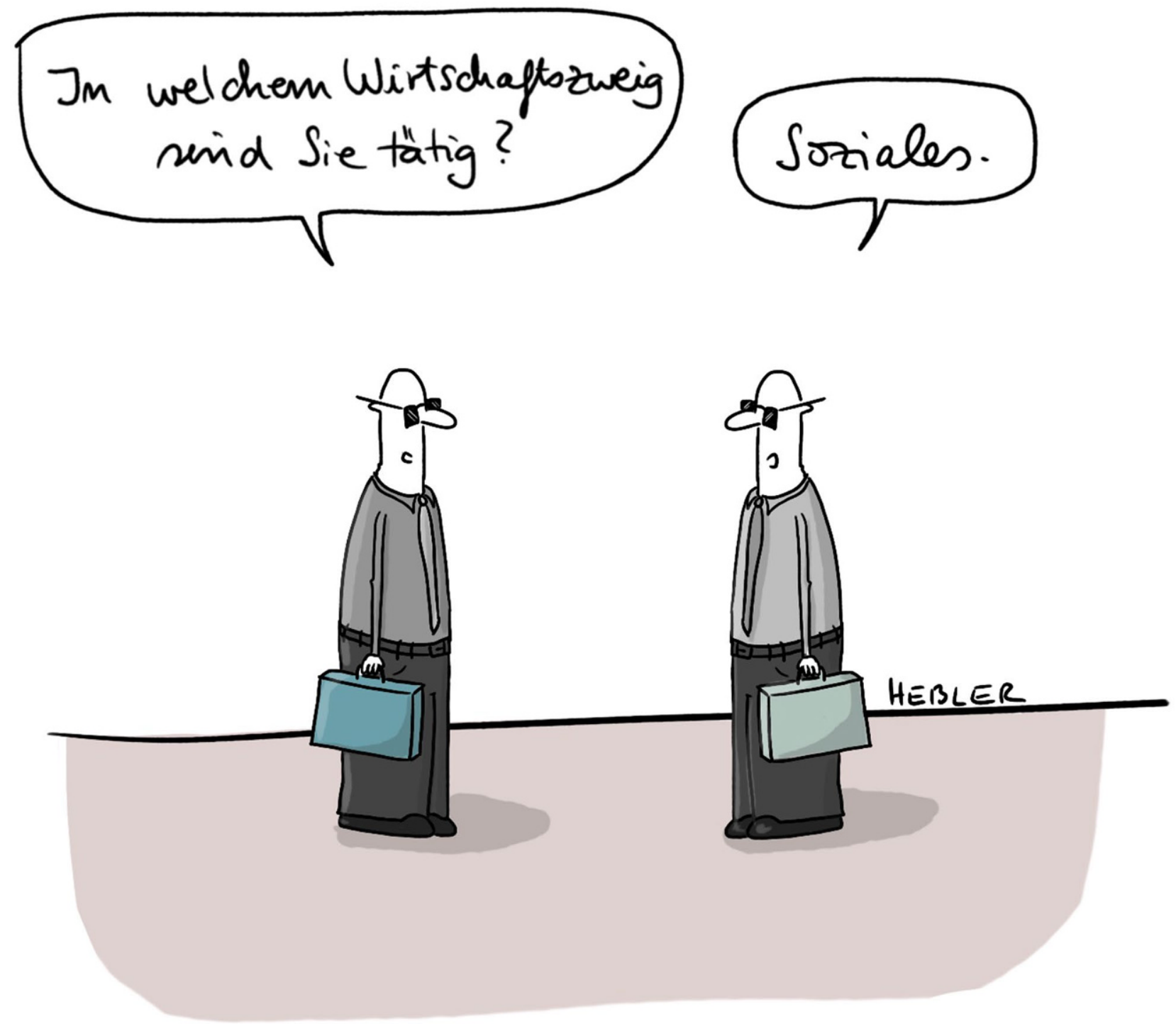

Diskrepanz begründet, die sich feststellen lässt im Verhältnis ihrer Grundsätze und ideologischen Wertorientierungen einerseits zu ihrer faktischen sozialpolitischen Funktion (Sozialwirtschaft) sowie zu den entsprechenden Strukturen und Organisationsformen (Sozialmanagement) andererseits. Außerdem wird das ideologische Selbstverständnis der W., ihre weltanschauliche Corporate Identity, u. a. durch das fachlich-professionelle Selbstverständnis ihrer Mitarbeiter (Professionalisierung, Soziale Berufe) z. T. überlagert, z. T. ersetzt“ (Bauer 2017, S. 1096).

Für Außenstehende erscheint die Landschaft der Wohlfahrtsverbände längst unübersichtlich geworden zu sein: Die Unterschiede zwischen den (noch) relativ „reichen“, christlich geprägten und den anerkannt „armen“ Wohlfahrtsverbänden und damit einhergehende (un- abhängige) Handlungsspielräume spielen dabei ebenso eine Rolle wie die Vielfalt der Organisationsgestaltung. Während die einen auf kommunaler, Landes- und Bundesebene in einer Dachorganisation präsent und in die jeweiligen sozialpolitischen Machtverhältnisse eingebunden sind und dabei mitunter komplexe Trägernetzwerke aufgebaut haben, über die sie ihre Dienstleistungen erbringen, bieten sich andere - vor allem der Paritätische - als Dachverband für eine Vielzahl heterogener kleiner(er) Vereine und Verbände an. Diese wiederum sind auf ein solches Dach angewiesen, um den heutigen Anforderungen an das Sozialmanagement gerecht zu werden und auf dem konkurrierenden Markt der Sozialwirtschaft Zugang zu öffentlichen und privaten Zuwendungen zu erhalten. Die Differenzen hinsichtlich der historischen Entwicklung, dem wohlfahrtsverband- 


\section{Extrablick: Wohlfahrtsverbände in der Legitimationskrise}

lichen Selbstverständnis und der Organisationsstruktur erschwert evidenzbasierte Vergleiche zwischen den Verbänden enorm.

Vor dem Hintergrund einer überschaubaren Datenlage wird es auch diesem Themenschwerpunkt nur gelingen, einige Schlaglichter auf die Spannungsfelder rund um die Wohlfahrtsverbände zu werfen, die nicht zuletzt aufgrund ihrer Geschichte, Organisation und gesellschaftspolitischer Position höchst unterschiedlich sind.

Zum Auftakt werden die Verbände durch Thomas Rauschenbach, Felix Berth und Matthias Hoffjan vermessen, indem sie die Personalentwicklung der letzten drei Jahrzehnte statistisch rekonstruieren und versuchen, sie ins Verhältnis zur Personalentwicklung in anderen Branchen und Sektoren zu setzen. Die Autoren betonen, dass es sich dabei nur um eine Annäherung handeln kann, da es an umfassenden und aussagekräftigen Daten zur vielfältigen Struktur der Beschäftigungsverhältnisse und ehrenamtlichen Mitarbeiter_innen in den Mitgliedsorganisationen fehle, was angesichts der Bedeutung der Wohlfahrtsverbände für den deutschen Arbeitsmarkt erstaunt.

Anschließend sondieren Barbara Lochner und Jörg Altmann vor dem Hintergrund öffentlicher Debatten um die Angemessenheit von Vergütungen des Managements sozialer Organisationen rechtliche und sozioökomische Hintergründe von Gemeinnützigkeit. Im Kern gehen sie der Frage nach, ob und inwiefern Wohlfahrtsverbände und andere sozial-gemeinnützige Organisationen im Unterschied zur freien Wirtschaft ihre Geschäftstätigkeit in besonderer Weise legitimieren müssen. Dabei wird deutlich, wie schwer es ist, den „Wert“ des Managements in sozialen Organisationen und insbesondere in Wohlfahrtsverbänden zu bestimmen oder ins Verhältnis zu wirtschaftlichen Unternehmen zu setzen. Sie plädieren für transparente Aushandlungsprozesse auf rechtlich abgesicherter Basis, welche für die Glaubwürdigkeit und letztlich auch für den Erhalt der Organisationen von entscheidender Bedeutung sind.

Nach dieser Auseinandersetzung mit den wirtschaftlichen Handlungsspielräumen von Wohlfahrtsverbänden beleuchtet Martina Messan den Anspruch advokatorischer Interessenvertretung, der Teil des wohlfahrtsverbandlichen Selbstverständnisses ist. Sie fasst dafür empirische Ergebnisse aus ihrer Promotionsforschung zusammen und arbeitet heraus, auf welche Weise die Verbände ihrem Anspruch auf eine Interessenvertretung bzw. auf eine Anwaltschaft marginalisierter Gruppen entsprechen können. Diesbezüglich regt sie eine kritische Selbstreflexion der Verbände an.

Die Perspektive von Martina Messan auf Wohlfahrtsverbände als anwaltschaftliche Vertretung marginali- sierter Gruppen wird ergänzt durch den Blick, den Karla Brennecke-Roos und Michael Franz im Interview von Helmuth Schweitzer auf Wohlfahrtsverbände werfen. Die beiden langjährigen ehemaligen Vorstandsmitglieder der AWO in Essen und des Bezirksverbands Niederrhein klären über die Bedeutung des Ehrenamts als Brückenbauerin zwischen Gemeinwesen, Politik und Wohlfahrt und die Relevanz sozialpolitischer Grundhaltungen für die Ausgestaltung konkreter sozialer Praxis auf. In ihrem persönlichen historischen Rückblick vermitteln sie, wie sich verbandliche Teilhabemöglichkeiten und die ehrenamtliche Verantwortung für die nachhaltige Existenzsicherung der Mitgliedsorganisationen und der Beschäftigten unter dem gewachsenen Druck marktwirtschaftlicher Konkurrenz um Refinanzierung ihrer vielfältigen Sozialen Arbeit aus öffentlichen Zuschüssen in den letzten beiden Jahrzehnten verändert haben. Abschließend stellt Dirk Kratz die komplexen Anforderungen an das Management in sozial-gemeinnützigen Organisationen in Verbindung zur Wohlfahrt aus einer Innenperspektive zusammen. Sein Kommentar weist dabei nicht nur auf die engen Handlungsgrenzen und die damit verbundenen Risiken hin, sondern geht ebenfalls auf die politischen Prozesse ein, die den Alltag von Führungskräften der Sozialen Arbeit prägen. Dabei ist die Arbeit auf der Verbandsebene von teilweise existenzieller Bedeutung.

In der Gesamtschau der Beiträge ist hervorzuheben, dass in den letzten Jahrzehnten die politischen, sozialwirtschaftlichen und personellen Rahmenbedingungen der freien Wohlfahrtspflege zunehmend vielfältiger gewordenen sind. Unter den Dächern der Wohlfahrtsverbände hat sich eine komplexe Dienstleistungsinfrastruktur etabliert, die gekennzeichnet ist durch einen immensen Zuwachs an sozialversicherungspflichtigen Beschäftigten, die in weit ausdifferenzierten Tätigkeitsbereichen aktiv sind. Die Mitgliedsorganisationen sind geprägt durch je spezifische Selbstverständnisse, differente Managementkompetenzen und Führungskulturen, unterschiedliche Formen der gesellschaftlichen, politischen sowie medialen Anerkennung und Unterstützung. Sie verfügen über ein unterschiedliches Maß an institutionalisierter Macht. Zugleich verlieren ideologische oder gar parteipolitische Ausrichtungen an Bedeutung für die Gestaltung der Sozialen Arbeit in den Verbänden. Diese Entwicklungen, aber auch ihre gesellschaftspolitischen Auswirkungen auf die Perspektiven für ein zukunftsfähiges und sozial gerechtes Zusammenleben unter der Bedingung begrenzter und umkämpfter Ressourcen sind noch bei Weitem nicht angemessen in der Öffentlichkeit reflektiert, geschweige denn wissenschaftlich erforscht. 
$\mathrm{Zu}$ den Forschungsdesiderata im Bereich der Wohlfahrtsverbändeforschung gehört insbesondere eine differenzierte empirische Analyse der Finanzierungsgrundlagen und ihrer Auswirkungen auf die Gestaltung von Beschäftigungsverhältnissen und Dienstleistungen. Welche Rolle spielen etwa zeitlich begrenzte „Modellprojekte“, die im Rahmen von ein- bis dreijähren Förderprogrammen staatlicher und privater Geldgeber (Stiftungen) unter „innovativen“ Projektüberschriften akquiriert werden? Befördern ökonomische Zwänge eine sogenannte „Projektitis“ bei den Verbänden, in deren Rahmen eigentlich grundständige Aufgaben der Verbandsarbeit richtlinienkompatibel zu Projekten umgedeutet werden (vgl. Schweitzer 2010)? Wie frei sind die Verbände, eingeworbene Mittel zur „Querfinanzierung “ zu nutzen und damit eigene Schwerpunkte zu setzen - z. B. für Bereiche, die strukturell unterfinanziert, bildungs- und sozialpolitisch aber dringend geboten sind, weil sie niedrigschwellige Leistungen für junge, arme, mehrfach diskriminierte Bevölkerungsgruppen sichern (vgl. Schweitzer 2006)? Was sind die Hintergründe sowie Auswirkungen der Umwandlung bzw. Ausgründung einzelner Abteilungen der Wohlfahrtsverbände als Töchter-Unternehmen in Form rechtlich eigenständiger gemeinnütziger $\mathrm{GmbH}$ ? Wie entwickeln sich vor diesem Hintergrund die Mitgliedsorganisationen als Orte des sozialen Engagements und des Ehrenamts? Welchen Einfluss haben diese Veränderungen auf die Gestaltung des Managements?

Eine gezielte Analyse solcher Trends würde die Basis bereiten für eine fundierte Reflexion und transparente Diskussion der gesellschaftlichen Rolle und Funktion von Wohlfahrtsverbänden. Mit Blick auf die Größe, die Veränderungen und die Bedeutung von Wohlfahrtsverbänden erscheint dies überfällig.

Eingegangen. 20. September 2021

Angenommen. 20. September 2021

Funding. Open Access funding enabled and organized by Projekt DEAL.

Open Access. Dieser Artikel wird unter der Creative Commons Namensnennung 4.0 International Lizenz veröffentlicht, welche die Nutzung, Vervielfältigung, Bearbeitung, Verbreitung und Wiedergabe in jeglichem Medium und Format erlaubt, sofern Sie den/die ursprünglichen Autor(en) und die Quelle ordnungsgemäß nennen, einen Link zur Creative Commons Lizenz beifügen und angeben, $o b$ Änderungen vorgenommen wurden.

Die in diesem Artikel enthaltenen Bilder und sonstiges Drittmaterial unterliegen ebenfalls der genannten Creative Commons Lizenz, sofern sich aus der Abbildungslegende nichts anderes ergibt. Sofern das betreffende Material nicht unter der genannten Creative Commons Lizenz steht und die betreffende Handlung nicht nach gesetzlichen Vorschriften erlaubt ist, ist für die oben aufgeführten Weiterverwendungen des Materials die Einwilligung des jeweiligen Rechteinhabers einzuholen.

Weitere Details zur Lizenz entnehmen Sie bitte der Lizenzinformation auf http://creativecommons.org/licenses/by/4.0/deed.de.

\section{Literatur}

Bauer, R. (2017). Wohlfahrtsverbände. In D. Kreft \& I. Mielenz (Hrsg.), Wörterbuch Soziale Arbeit. Aufgaben, Praxisfelder, Begriffe und Methoden der Sozialarbeit und Sozialpädagogik (8. Aufl. S. 1094-1098). Weinheim, Basel: Beltz Juventa.

Schweitzer, H. (2006). Sozialpleiten - nur Pech und Pannen? Sozial Extra, 30(1), 8-13.

Schweitzer, H. (2010). Das „System Modellprojekte“. Von der nachhaltigen Wirkung eines Alibis in der Sozial- und Bildungspolitik. Sozial Extra, 34(7/8), 14-19. 